\title{
Elderly Patients with Advanced Non-Small Cell Lung Cancer: What Treatment?
}

\author{
Antonio Rossi*
}

Division of Medical Oncology, “S.G. Moscati” Hospital, Avellino, Italy

\begin{abstract}
Advanced non-small cell lung cancer (NSCLC) in elderly patients is an increasingly common problem which the practitioner of oncology must face. There is no consensus on the cut-off age for defining the elderly. However, 70 years may be the most appropriate because the incidence of age-related changes starts to increase after this boundary. Important concerns in evaluating the treatment of elderly patients are the presence of comorbidities and the progressive physiologic reduction of hepatic, renal and bone-marrow functions which could have a negative impact on the degree of toxicity. To individualize treatment choice within a group of elderly NSCLC patients of the same chronological age, it would be important to perform a comprehensive geriatric assessment (CGA) which would allow to subdivide elderly patients into three main categories: fit, pre-frail and frail. Fit older patients have similar prognosis and a similar treatment tolerance and outcome compared to their younger counterparts. On the other hand, pre-frail patients experience significant treatment related toxicity and usually are offered a single-agent chemotherapy whose choice should take into account the expected toxicity profile of the agent, pharmacokinetics, organ function and co-morbidities. For the third category of patients only best supportive care or individualized approaches are recommended.
\end{abstract}

Overall, only prospective trials, specifically addressed to elderly NSCLC patients selected through an adequate CGA at baseline, let us opt for the best treatment to be administered to each elderly patient.

Keywords: NSCLC, comorbidities, CGA, elderly, chemotherapy, targeted agents.

\section{INTRODUCTION}

Lung cancer is the leading cause of cancer-related mortality in both men and women [1], with about 1.61 million new diagnoses and 1.38 million deaths worldwide in 2008 [2]. Non-small cell lung cancer (NSCLC), including squamous carcinoma, adenocarcinoma and undifferentiated large cell carcinoma, accounts for more than $80 \%$ of new lung cancer diagnoses [3]. Unfortunately, at the time of diagnosis, the majority of patients have advanced disease, for which a systemic, palliative treatment is the primary therapeutic option. Considering that $47 \%$ of all lung cancers are diagnosed in patients older than 70 years $(14 \%$ in patients older than 80 years) [4], advanced NSCLC in elderly patients is an increasingly common problem which the practitioner of oncology must face.

Establishing the exact age, i.e. the biological age, is still difficult nowadays due to the lack of adequate laboratory tests and tools. Thus, the chronological age is the only indicator we have in defining the elderly, and 70 years may be the most appropriate boundary because the incidence of age-related changes starts to increase after this cut-off age [5]. Important concerns in evaluating the treatment of elderly patients are the presence of comorbidities and the progressive physiologic reduction of hepatic, renal and bonemarrow functions which could have a negative impact on the degree of toxicity. The most important co-existing

\footnotetext{
*Address correspondence to this author at the Division of Medical Oncology, "S.G. Moscati" Hospital, Contrada Amoretta, 8, 83100 Avellino, Italy; Tel: + 390825 203573; Fax: + 390825 203556;

E-mail: arossi_it@yahoo.it
}

pathologies in lung cancer patients are cardiovascular and pulmonary diseases, common among heavy smokers. Drugs used to treat these comorbidities may interact with anticancer agents, thus exacerbating their toxicity. In fact, many anticancer drugs are metabolized by cytocrome P450 enzymes, which can be induced or inhibited by many commonly prescribed medications. Therefore, drug interactions can be a particular concern in polypharmacy [6]. Hence, the presence of these medical and physiological challenges make the selection of their optimal treatment daunting, a reason for which these patients are often under-treated [7].

Taking into account these considerations, in order to individualize treatment choice within a group of elderly NSCLC patients of the same chronological age, it would be important to perform a comprehensive geriatric assessment (CGA). This procedure evaluates the patients' global and functional status, in order to improve treatment decisions and outcomes. The CGA estimates a patient's functional status, the presence of comorbidities, mental status and emotional condition, social support, the nutritional status, polypharmacy and the presence or absence of geriatric syndromes, thus allowing to subdivide elderly patients into three main categories: fit, pre-frail and frail [8].

To date, several randomized phase III trials specifically addressed to advanced NSCLC elderly patients were performed and several others are ongoing. This review will assess today's standard of care for this group of patients.

\section{TREATMENT STRATEGIES}

Retrospective charts analyses evaluated the impact of therapy in terms of activity, efficacy and toxicity on 
advanced NSCLC elderly patients, arising therapeutic hypotheses to be evaluated prospectively in this subset of patients. Several therapeutic approaches have been investigated for the treatment of elderly patients and many results are already available.

\section{Single-Agent Chemotherapy}

This approach has been addressed by two randomized phase III studies [9, 10]. The Elderly Lung Cancer Vinorelbine Italian Study (ELVIS) was the first randomised phase III trial ever performed in advanced NSCLC patients aged $\geq 70$ years. A total of 191 elderly, with an Eastern Cooperative Oncology Group (ECOG) performance status (PS) $\leq 2$, were randomised to single-agent vinorelbine $(\mathrm{n}=$ 76), at the dose of $30 \mathrm{mg} / \mathrm{m}^{2}$ day 1 and 8 every 3 weeks, plus best supportive care (BSC) or BSC alone $(n=78)$. Quality of life (QoL) was the primary endpoint of the study. Vinorelbine-treated patients scored better than control patients on QoL functioning scales, and they reported fewer lung cancer-related symptoms but reported worse toxicityrelated symptoms. Vinorelbine improved median overall survival (OS) which was 27 versus 21 weeks reported by BSC alone $(p=0.04)$. The relative hazard ratio (HR) for death for vinorelbine-treated patients was $0.65 \quad(95 \%$ confidence interval [CI] 0.45-0.93) [9]. The ELVIS study represents a landmark regarding the feasibility and palliative role of chemotherapy in elderly patients with advanced NSCLC. The other randomized phase III trial compared two single-agent, vinorelbine versus docetaxel. The primary endpoint was OS. A total of 182 patients, aged $\geq 70$ years and with a PS $\leq 2$, were randomized to receive docetaxel ( $\mathrm{n}$ $=88$ ), administered at $60 \mathrm{mg} / \mathrm{m}^{2}$ day 1 every 3 weeks, or vinorelbine $(\mathrm{n}=91)$, at the dose of $25 \mathrm{mg} / \mathrm{m}^{2}$ day 1 and 8 every 3 weeks. Docetaxel reported a better OS (14.3 versus 9.9 months, respectively; HR $0.780 ; 95 \%$ CI $0.561-1.085 ; \mathrm{p}$ $=0.138)$, improved progression-free survival (PFS) $(5.5$ versus 3.1 months; HR 0.606, 95\% CI 0.450-0.816; $\mathrm{p}<$ $0.001)$ and response rate $(22.7 \%$ versus $9.9 \% ; \mathrm{p}=0.019)$ versus vinorelbine, but was associated with more grade 3-4 neutropenia $(82.9 \%$ for docetaxel; $69.2 \%$ for vinorelbine; $\mathrm{p}=$ 0.031). In terms of global QoL, no significant difference was observed between the two arms (odds ratio [OR] 1.30; $95 \%$ CI 0.80-2.11). Docetaxel was associated with improvement in the overall symptom score compared to vinorelbine (OR 1.86; 95\% CI 1.09-3.20) [10] (Table 1).

Globally, these two trials established that a thirdgeneration single-agent chemotherapy represents the standard of care for unselected advanced NSCLC elderly patients.

\section{Non-Platinum-Based Regimens}

Vinorelbine plus gemcitabine is the most studied nonplatinum-based regimen investigated in this setting. Also in this case, the randomized phase III trials addressing this issue were two in which single-agent therapy was compared with the gemcitabine plus vinorelbine doublet. The first trial investigated gemcitabine $1200 \mathrm{mg} / \mathrm{m}^{2}$ plus vinorelbine 30 $\mathrm{mg} / \mathrm{m}^{2}$ versus vinorelbine $30 \mathrm{mg} / \mathrm{m}^{2}$ alone $(\mathrm{n}=120$ patients, 60 per each arm), given on day 1 and 8 every 3 weeks. The patients enrolled were aged $\geq 70$ years and with a PS $\leq 2$. The primary endpoint was OS. This trial was closed early due to an interim analysis showing a survival advantage for the doublet over the single-agent (OS: 29 versus 18 weeks, respectively; $\mathrm{p}<0.01)$. The 1 -year survival was $30 \%$ and $18 \%$, respectively with a HR for death of 0.48 (95\% CI 0.29 $0.79)$. The response rate favoured the doublet too, with $22 \%$ versus $15 \%$, respectively. A total of $14(26 \%)$ patients in the doublet arm showed temporary symptom relief during the treatment, compared with $8(15 \%)$ patients treated with single-agent. While almost $60 \%$ and $40 \%$ of patients did not show impairment of the QoL score during the treatment, respectively [11]. The second and the largest randomized phase III trial ever performed in the elderly NSCLC patients was called MILES (Multicenter Italian Lung Cancer in the Elderly Study). The trial accrued 698 patients aged $\geq 70$ years and with PS $\leq 2$ showing that the combination of vinorelbine $\left(25 \mathrm{mg} / \mathrm{m}^{2}\right)$ plus gemcitabine $\left(1000 \mathrm{mg} / \mathrm{m}^{2}\right)$ was no more effective than single-agent vinorelbine $\left(30 \mathrm{mg} / \mathrm{m}^{2}\right)$ or gemcitabine $\left(1200 \mathrm{mg} / \mathrm{m}^{2}\right)$ given on day 1 and 8 , every 3 weeks. The primary endpoint was OS between each singleagent and doublet, the trial was not designed to compare directly the two single-agent arms. The median age of enrolled patients was 74 years with 275 patients (39\%) aged 75 years or older. The OS was 36, 28 and 30 weeks, and the probability of being alive at 1 -year of $38 \%, 28 \%$ and $30 \%$, for vinorelbine, gemcitabine or their combination, respectively. The HR for death was 1.17 (95\% CI 0.95-1.44) for the combination treatment versus vinorelbine and 1.06 (95\% CI 0.86-1.29) for the combining regimen versus gemcitabine. Although QoL was similar across the three treatment arms, the combination treatment was slightly more toxic than the two drugs given singly. In fact, combination chemotherapy resulted in higher thrombocytopenia and hepatic toxicity compared to single-agent vinorelbine, and higher neutropenia, vomiting, fatigue, cardiac toxicity and

Table 1. Results from Phase III Trials with Single-Agent Therapy in the Treatment of Advanced Non-Small-Cell Lung Cancer Patients Aged $\geq 70$ Years

\begin{tabular}{|c|c|c|c|c|c|c|}
\hline Author & Regimen & No.pts & RR (\%) & PFS (Months) & OS (Months) & QoL \\
\hline ELVIS, 1999 [9] & $\begin{array}{c}\text { Vinorelbine } \\
v s \\
\text { BSC }\end{array}$ & $\begin{array}{l}76 \\
78\end{array}$ & $\begin{array}{l}20 \\
\mathrm{NA}\end{array}$ & NR & $\begin{array}{l}6.5 \\
4.8\end{array}$ & Vinorelbine better in QoL functioning scales \\
\hline Kudoh, 2006 [10] & $\begin{array}{c}\text { Vinorelbine } \\
\qquad s \\
\text { Docetaxel }\end{array}$ & $\begin{array}{l}91 \\
88\end{array}$ & $\begin{array}{l}9.9 \\
22.7\end{array}$ & $\begin{array}{l}3.1 \\
5.4\end{array}$ & $\begin{array}{r}9.9 \\
14.3\end{array}$ & OR 1.30 \\
\hline
\end{tabular}

No.pts: number of patients; RR: response rate; PFS: progression-free survival; OS: median survival; QoL: quality of life; ELVIS: Elderly Lung cancer Vinorelbine Italian Study; NA: not applicable; NR: not reported; OR: odds ratio. 
Table 2. Results from Phase III Trials with Not Platinum-Based Regimens in the Treatment of Advanced Non-Small-Cell Lung Cancer Patients Aged $\geq 70$ Years

\begin{tabular}{|c|c|c|c|c|c|c|}
\hline Author & Regimen & No.pts & RR (\%) & $\begin{array}{c}\text { TTP } \\
\text { (Months) }\end{array}$ & $\begin{array}{c}\text { OS } \\
\text { (Months) }\end{array}$ & QoL \\
\hline Frasci, $2000[11]$ & $\begin{array}{c}\text { VNR } \\
v s \\
\text { VNR + GEM }\end{array}$ & $\begin{array}{l}60 \\
60\end{array}$ & $\begin{array}{l}15 \\
22\end{array}$ & NR & $\begin{array}{l}4.2 \\
6.7\end{array}$ & $\begin{array}{l}60 \% \text { and } 40 \% \text { of patients did not show impairment of the QoL } \\
\text { score in doublet and single-agent therapy, respectively }\end{array}$ \\
\hline Gridelli, 2003 [12] & $\begin{array}{c}\text { VNR } \\
\text { or } \\
\text { GEM } \\
v s \\
\text { VNR + GEM }\end{array}$ & $\begin{array}{l}233 \\
232\end{array}$ & $\begin{array}{l}16 \\
21\end{array}$ & $\begin{array}{r}4.5 \\
4.25 \\
4.75\end{array}$ & $\begin{array}{l}6.5 \\
6.9\end{array}$ & QoL was similar across the three treatment arms \\
\hline
\end{tabular}

No.pts: number of patients; RR = response rate; TTP: time to progression; OS: median survival; QoL: quality of life; VNR: vinorelbine; GEM: gemcitabine; NR: not reported

constipation compared to single-agent gemcitabine [12] (Table 2).

Based on these last observations, single-agent chemotherapy was confirmed to be a reasonable treatment choice and certainly the standard for comparison in unselected elderly patients with advanced NSCLC.

\section{Platinum-Based Regimens}

This issue has been firstly addressed in retrospective analyses of large randomised trials which had no age limit for enrolling patients. The treatment outcomes, coming from these randomized phase 3 trials, were similar between patients younger and older than 70 years but with a small increase in toxicity in the elderly, suggesting that advanced age alone should not preclude this subset of patients to platinum-based chemotherapy. Nevertheless, the elderly patients enrolled in these trials are not representative of the real elderly population but rather of a small subgroup considered by investigators to be eligible for aggressive treatments [13]. Therefore, there is a need for prospective clinical trials of platinum-based chemotherapy with inclusion criteria limited to the elderly population. At the time of writing, only two phase III prospective randomized trials with carboplatin-based regimens addressed to elderly NSCLC patients are available with preliminary results.

A randomized phase 3 trial compared single-agent gemcitabine $\left(1150 \mathrm{mg} / \mathrm{m}^{2}\right)$ or vinorelbine $\left(30 \mathrm{mg} / \mathrm{m}^{2}\right)$ on day 1 and 8, every 3 weeks versus carboplatin (area under curve [AUC] 6 on day 1) plus paclitaxel $\left(90 \mathrm{mg} / \mathrm{m}^{2}\right.$ on day 1,8 and 15) every 4 weeks. Primary endpoint was OS. A total of 451 patients aged from 70 to 89 years, with PS 0-2, were randomized. The OS was significantly longer for patients treated with combination chemotherapy (10.4 versus 6.2 months; HR 0.639, 95\% CI 0.515-0.792; p < 0.0001). The 1 year survival was $45.1 \%$ for the doublet and $26.9 \%$ for the single-agent with a median PFS of 6.1 versus 3.0 months, respectively. Response rate was $29 \%$ versus $10.9 \%$, respectively. However, grade 3-4 hematologic toxicities and treatment-related deaths were significantly more frequent in patients treated with carboplatin and paclitaxel as compared to single-agent gemcitabine or vinorelbine [14]. Another phase III randomized trial enrolled 181 patients, aged $\geq 70$ years and with $\mathrm{PS} \leq 2$, to receive two doublets, carboplatin (AUC 5 on day 1) plus gemcitabine $\left(1250 \mathrm{mg} / \mathrm{m}^{2}\right.$ on day 1 and 8 ) or paclitaxel $\left(175 \mathrm{mg} / \mathrm{m}^{2}\right.$ on day 1$)$, every 3 weeks for a maximum of 4 cycles. These doses were similar to those generally administered in younger patients. The main endpoint was QoL. Overall, grade 3-4 toxicity occurred in $75 \%$ and $60 \%$ of patients treated with carboplatin plus gemcitabine or paclitaxel, respectively. The confirmed response rate was $27 \%$ and $19 \%$ with a median PFS of 4.7 and 4.5 months and a OS of 8.6 and 6.9 months, respectively. Mean global QoL score at baseline did not differ between both arms without any statistically difference at 18 weeks analysis [15]. The number of QoL responders $(12 \%$ and $5 \%$ in carboplatin plus gemcitabine or paclitaxel, respectively) was not significantly different. A CGA was also carried out with $38 \%$ and $25 \%$ of patients enrolled in gemcitabine-based arm and paclitaxel-based arm reporting $\geq$ 2 comorbidities, respectively. Almost half of patients had limitations in instrumental activities of daily living (IADL), and more than a quarter had abnormal depression scores. There were no significant interactions between CGA scores and treatment (Table 3 ).

The results coming from these two trials, in which carboplatin-based doublets with doses typically used for adult patients were administered, seem to call for studies investigating platinum-based regimens with doses finding for elderly. In this view, several published phase II studies of combination chemotherapy based on modified schedules of carboplatin (low-dose or weekly administration) have shown interesting activity and good tolerability [13]. However, of interest is also the exploration of innovative schedules and attenuated doses of cisplatin that would be more suitable in the elderly. Several small phase II trials tested the combination of third-generation cytotoxic agents with cisplatin in modified schedules or attenuated doses, in the quest for active and well tolerated treatment regimens [13]. A phase I/II randomised trial, MILES-2P, evaluated the feasibility of cisplatin at attenuated doses combined with gemcitabine or vinorelbine in patients with advanced NSCLC aged $\geq 70$ years and with PS $0-1$. Cisplatin was feasible and active at $60 \mathrm{mg} / \mathrm{m}^{2}$ on day 1 , with gemcitabine $\left(1000 \mathrm{mg} / \mathrm{m}^{2}\right.$ on day 1 and 8$)$ and at $40 \mathrm{mg} / \mathrm{m}^{2}$ on day 1 , with vinorelbine $\left(25 \mathrm{mg} / \mathrm{m}^{2}\right.$ on day 1 and 8$)$, every 3 weeks. With the former combination, $83.3 \%$ of patients were treated without unacceptable toxicity, response rate was $43.5 \%$ (95\% CI 30.6-56.8), median PFS and OS were 25.3 and 43.6 weeks, respectively. With the latter combination, $82 \%$ of patients were treated without unacceptable toxicity, response 
Table 3. Results from Phase III Trials with Platinum-Based Regimens in the Treatment of Advanced Non-Small-Cell Lung Cancer Patients Aged $\geq 70$ Years

\begin{tabular}{|c|c|c|c|c|c|c|}
\hline Author & Regimen & No.pts & RR (\%) & PFS (Months) & OS (Months) & QoL \\
\hline Quoix, 2010 [14] & $\begin{array}{c}\text { GEM or VNR } \\
v s \\
\text { CBDCA + PAC }\end{array}$ & $\begin{array}{l}226 \\
225\end{array}$ & $\begin{array}{l}10.9 \\
29.5\end{array}$ & $\begin{array}{l}3.0 \\
6.1\end{array}$ & $\begin{array}{l}6.2 \\
10.3\end{array}$ & NR \\
\hline Biesma, 2011 [15] & $\begin{array}{c}\mathrm{CBDCA}+\mathrm{GEM} \\
v s \\
\mathrm{CBDCA}+\mathrm{PAC}\end{array}$ & $\begin{array}{l}90 \\
91\end{array}$ & 19 & $\begin{array}{l}4.7 \\
4.5\end{array}$ & $\begin{array}{l}8.6 \\
6.9\end{array}$ & $\begin{array}{c}\text { QoL score without any difference from } \\
\text { baseline to week } 18\end{array}$ \\
\hline
\end{tabular}

No.pts: number of patients; RR: response rate; PFS: progression-free survival; OS: median survival; QoL: quality of life; CBDCA: carboplatin; GEM: gemcitabine; VNR : vinorelbine; PAC: paclitaxel; NR: not reported.

rate was 36.1\% (95\% CI 24.2-49.4), median PFS and OS were 21.1 and 33.1 weeks, respectively. The combination of cisplatin plus gemcitabine, which provided a higher dose of cisplatin, deserved further investigation versus single-agent chemotherapy in this setting of patients [16].

These data seem to confirm that only modified platinumbased regimens specifically studied in the elderly population with no major comorbidities and a good PS (0-1) could be administered maintaining the efficacy and without worsening the toxicity.

\section{New Biological Drugs}

Advances in the understanding of lung cancer molecular abnormalities has led to the identification of genes involved in lung carcinogenesis which are being used as target for new biologic agents. Two pathways were particularly studied and provided specific inhibitors which are currently in the clinical practice for the treatment of advanced NSCLC patients: the epidermal growth factor receptor (EGFR) and the vascular endothelial growth factor (VEGF).

The EGFR pathway can be blocked by two small molecules, orally administered, tyrosine kinase inhibitors (TKIs), gefitinib and erlotinib, and by a monoclonal antibody, administered intravenously, cetuximab. Several retrospective analyses and prospective randomized phase 3 trials identified clinical (never smoker status, adenocarcinoma histology, female sex, and Asian ethnicity) and biological (EGFR amplification, EGFR protein expression, EGFR mutations, and K-ras mutations) factors which seem to be predictive of activity to EGFR-TKIs. To date, the only factor resulted clearly predictive of activity for EGFR-TKIs is the presence of EGFR mutations. Exon 19 deletions and exon 21 L858R substitution account for about $85 \%$ of all EGFR mutations in NSCLC [17]. Gefitinib is currently licensed for the treatment of adult patients with locally advanced or metastatic NSCLC with activating mutations of EGFR. Erlotinib is licensed as monotherapy for maintenance treatment in patients with locally advanced or metastatic NSCLC with stable disease after 4 cycles of standard platinum-based first-line chemotherapy and for the treatment of patients with locally advanced or metastatic NSCLC after failure of at least one prior chemotherapy regimen regardless to clinical and/or biologic factors.

Gefitinib was investigated specifically also in elderly patients unselected for any clinical or molecular factors. A phase II randomized trial compared gefitinib $(\mathrm{n}=97 ; 250 \mathrm{mg}$ orally, daily) to vinorelbine $\left(\mathrm{n}=99 ; 30 \mathrm{mg} / \mathrm{m}^{2}\right.$ day 1 and 8 , every 3 weeks) as first-line treatment of advanced NSCLC patients aged $\geq 70$ years and with $\mathrm{PS} \leq 2$. The primary endpoint was PFS. The HRs for gefitinib versus vinorelbine were 1.19 (95\% CI 0.85-1.65) for PFS and 0.98 (95\% CI $0.66-1.47)$ for OS. The response and disease control rates were $3.1 \%$ (95\% CI $0.6-8.8)$ and $43.3 \%$, for gefitinib, and $5.1 \% \quad(95 \%$ CI $1.7 \quad 11.4)$ and $53.5 \%$, for vinorelbine, respectively. Overall QoL and pulmonary symptoms improvement rates were $24.3 \%$ and $36.6 \%$, for gefitinib, and $10.9 \%$ and $31 \%$, for vinorelbine, respectively. Gefitinib was better tolerated with fewer treatment-related grade 3 to 5 adverse events (12.8\%) than with vinorelbine (41.7\%) [18].

Also erlotinib, at the dose of $150 \mathrm{mg}$ orally, daily, was investigated in 80 unselected elderly patients ( $\geq 70$ years) with previously untreated advanced NSCLC reporting a response rate of $10 \%$ with a stable disease of $41 \%$. There was a symptoms improvement (dyspnoea, cough, fatigue, pain) with an OS of 10.9 months. Rash and diarrhoea were the most common toxicities occurring respectively in $81 \%$ and $69 \%$ of the patients [19]. A randomized phase II trial compared oral vinorelbine $\left(60 \mathrm{mg} / \mathrm{m}^{2}\right.$ day 1 and 8 every 3 weeks for the first cycle and than $80 \mathrm{mg} / \mathrm{m}^{2}$ ) to erlotinib as first-line therapy of unselected patients aged $\geq 70$ years. Preliminary results reported on 77 patients showed a response rate of $21.6 \%$ with erlotinib and $12.8 \%$ with vinorelbine and no differences in terms of time to progression between the two arms (4.4 versus 3.9 months, respectively; $p=0.6069)$. The most common treatmentrelated toxicities were skin rash and diarrhoea with erlotinib, and diarrhoea and nausea with vinorelbine [20].

The monoclonal antibody cetuximab was investigated in the treatment of elderly patients, too. A phase II trial, the CALC1-E (Cetuximab in Advanced Lung Cancer - Elderly) study, was designed to define the optimal combination of cetuximab $\left(400 \mathrm{mg} / \mathrm{m}^{2}\right.$ the first week as loading dose and than $250 \mathrm{mg} / \mathrm{m}^{2} /$ week) with gemcitabine $\left(1200 \mathrm{mg} / \mathrm{m}^{2}\right.$ day 1 and 8 , every 3 weeks), i.e. a concomitant (gemcitabine, for a maximum of 6 cycles, plus cetuximab until disease progression) or a sequential (gemcitabine, for a maximum of 6 cycles, followed by cetuximab) treatment strategy. The CALC1-E study reported a 1-year survival rate, the main endpoint, for the concomitant and sequential arms of $41.4 \%$ and $31 \%$, with a PFS of 3 and 4 months, OS of 6 and 9 months, respectively. The response rate was $10.3 \%$ in both arms with a similar mild toxicity profile. Haematological toxicity was not frequent. Skin toxicity was observed in 20 
patients $(69.0 \%)$ in the concomitant arm, and in 18 patients $(62.0 \%)$ in the sequential arm. Fatigue was common, and it was grade 3 in $6(20.7 \%)$ and 4 (13.8\%) patients, respectively. Although the rate of patients alive at 1-year was higher in the combination arm for elderly patients, no striking differences in efficacy were observed. However, in the sequential strategy, $34 \%$ of elderly patients were never able to start cetuximab as maintenance or second-line treatment. These results suggest that combining gemcitabine and cetuximab from the beginning of treatment is the optimal way to give all the patients the chance of having benefit from cetuximab [21].

Bevacizumab is a VEGF monoclonal antibody inhibitor currently registered in combination with chemotherapy for first-line treatment of advanced non-squamous NSCLC patients due to a higher incidence of pulmonary haemorrhage reported in squamous histology. There is a lack of prospective data about its use in the elderly population with results coming only from retrospective analyses. The ECOG 4599 was a randomized phase III trial comparing carboplatin, AUC 6, plus paclitaxel, $200 \mathrm{mg} / \mathrm{m}^{2}$ without or with bevacizumab, $15 \mathrm{mg} / \mathrm{kg}$, all drugs given on day 1 every 3 weeks. A subgroup analysis of the older patients $(n=224$ $\geq 70$ years) randomized in the ECOG 4599 study yielded a trend towards higher response rate $(29 \%$ versus $17 \%$; $\mathrm{p}=$ 0.067 ) and higher PFS (5.9 versus 4.9 months; $p=0.063$ ) in favour of the bevacizumab arm and no difference in OS (11.3 versus 12.1 months; $p=0.4$ ). However, the older patients experienced significant grade $\geq 3$ toxicities with the addition of bevacizumab, compared to the paclitaxel/carboplatin doublet. Seven treatment-related deaths were observed among elderly patients treated with the three-drug combination compared with only two deaths in the chemotherapy alone arm. Furthermore, older patients who received bevacizumab suffered more grade $\geq 3$ toxicities compared to their younger counterparts [22]. The AVAiL (AVAstin in Lung) was another phase III randomized trial addressed to non-squamous advanced NSCLC patients in which the combination of cisplatin, 80 $\mathrm{mg} / \mathrm{m}^{2}$ on day 1 , plus gemcitabine, $1250 \mathrm{mg} / \mathrm{m}^{2}$ on day 1 and 8 , every 3 weeks was administered alone or in combination with two different doses of bevacizumab, 7.5 or $15 \mathrm{mg} / \mathrm{kg}$, on day 1 , every 3 weeks. An exploratory not planned retrospective analysis of the 304 patients older than 65 years was performed also for the AVAiL study. Patients who received bevacizumab derived a clinically relevant improvement in PFS compared with placebo $(7.5 \mathrm{mg} / \mathrm{kg}$ bevacizumab: HR $0.71, ; \mathrm{p}=0.023 ; 15 \mathrm{mg} / \mathrm{kg}$ bevacizumab: HR $0.84 ; p=0.25$ ). Response rates were $40 \%, 29 \%$ and $30 \%$ in the $7.5 \mathrm{mg} / \mathrm{kg}$ bevacizumab, $15 \mathrm{mg} / \mathrm{kg}$ bevacizumab and placebo arms, respectively. OS was similar for each bevacizumab arm versus placebo $(7.5 \mathrm{mg} / \mathrm{kg}$ bevacizumab: HR 0.84; $\mathrm{p}=0.31 ; 15 \mathrm{mg} / \mathrm{kg}$ bevacizumab: HR $0.88 ; \mathrm{p}=$ 0.44 ). Only grade $\geq 3$ thrombocytopenia occurred more frequently with bevacizumab compared with placebo in patients aged $\geq 65$ years $(13 \%$ higher in the $7.5 \mathrm{mg} / \mathrm{kg}$ arm and $11 \%$ in the $15 \mathrm{mg} / \mathrm{kg}$ arm) than those aged $<65$ years. The incidence of other grade $\geq 3$ adverse events with bevacizumab was similar in older and younger patients [23].

Overall, to date, among the licensed new biologic agents for the treatment of advanced NSCLC, elderly patients whose tumour harbours an EGFR mutations have to be treated with TKIs, gefitinib in any line of treatment, and erlotinib only in previously pretreated patients. The role of bevacizumab in the treatment of elderly should be evaluated prospectively due to the contrasting results emerged from retrospective analyses.

\section{CONCLUSIONS}

In the clinical practice, the therapeutic approach to the elderly NSCLC patients should be drived by the EGFR mutation status. If an EGFR mutation is detected, the treatment has to be a TKI. In presence of an EGFR wild type or unknown because there are no sufficient cells for the determination, the chemotherapeutic approach should take into account the three categories in which elderly NSCLC patients should be subdivided. Fit older patients have similar prognosis and a similar treatment tolerance and outcome compared to their younger counterparts. On the other hand, pre-frail patients experience significant treatment related toxicity and are usually offered a single-agent palliative chemotherapy with an adequate BSC and specific clinical trials. The choice of the single-agent to administer should take into account the expected toxicity profile of the agent, pharmacokinetics, organ function and co-morbidities. For the third category of patients only BSC or individualized approaches are recommended. Unfortunately, this subdivision is difficult to apply due to the lack, in the every day's practice, of easy CGA to administer to the patients and to analyse by the caregivers. Thus, only prospective trials, specifically addressed to elderly NSCLC patients, who should be selected through an adequate CGA at baseline, let us select for the best treatment to each elderly patient.

\section{CONFLICT OF INTEREST}

Dr. Antonio Rossi states that he has no conflict of interest.

\section{REFERENCES}

[1] Jemal A, Siegel R, Ward E, et al. Cancer statistics, 2008. CA Cancer J Clin 2008; 58: 71-96.

[2] Ferlay J, Shin HR, Bray F, Forman D, Mathers C, Parkin DM. Estimates of worldwide burden of cancer in 2008: GLOBOCAN 2008. Int J Cancer 2010; 127: 2893-917.

[3] Govindan R, Page N, Morgensztern D, et al. Changing epidemiology of small-cell lung cancer in the United States over the last 30 years: analysis of the Surveillance, Epidemiologic, and End Results database. J Clin Oncol 2006; 24: 4539-44.

[4] Owonikoko TK, Ragin CC, Belani CP, et al. Lung cancer in elderly patients: an analysis of the surveillance, epidemiology, and end results database. J Clin Oncol 2007; 25: 5570-7.

[5] Balducci L. Geriatric oncology: challenges for the new century. Eur J Cancer 2000; 36: 1741-54

[6] Rossi A, Maione P, Gridelli C. Safety profile of platinum-based chemotherapy in the treatment of advanced non-small cell lung cancer elderly patients. Expert Opin Drug Saf 2005; 4: 1037-53.

[7] Fentiman IS, Tirelli U, Monfardini S, et al. Cancer in the elderly: why so badly treated? Lancet 1990; 335: 1020-22.

[8] Extermann M, Hurria A. Comprehensive geriatric assessment for older patients with cancer. J Clin Oncol 2007; 25: 1824-31.

[9] The Elderly Lung Cancer Vinorelbine Italian Study Group. Effects of vinorelbine on quality of life and survival of elderly patients with advanced non small cell lung cancer. J Natl Cancer Inst 1999; 91: 66-72.

[10] Kudoh S, Takeda K, Nakagawa K, et al. Phase III study of docetaxel compared with vinorelbine in elderly patients with advanced non-small-cell lung cancer: results of the West Japan Thoracic Oncology Group trial (WJOG 9904). J Clin Oncol 2006; 24: 3657-63. 
[11] Frasci G, Lorusso V, Panza N, et al. Gemcitabine plus vinorelbine versus vinorelbine alone in elderly patients with advanced nonsmall-cell lung cancer. J Clin Oncol 2000; 18: 2529-36.

[12] Gridelli C, Perrone F, Gallo C, et al. Chemotherapy for elderly patients with advanced non-small-cell lung cancer: the Multicenter Italian Lung Cancer in the Elderly Study (MILES) phase III randomized trial. J Natl Cancer Inst 2003; 95: 362-72.

[13] Gridelli C, Langer C, Maione P, Rossi A, Schild SE. Lung cancer in the elderly. J Clin Oncol 2007; 25: 1898-907.

[14] Quoix EA, Oster J, Westeel V, et al. Weekly paclitaxel combined with monthly carboplatin versus single-agent therapy in patients age 70 to 89: IFCT-0501 randomized phase III study in advanced non-small cell lung cancer (NSCLC). J Clin Oncol 2010; 28(15S): 5s (abstr 2).

[15] Biesma B, Wymenga ANM, Vincent A, et al. Quality of life, geriatric assessment and survival in elderly patients with nonsmall-cell lung cancer treated with carboplatin-gemcitabine or carboplatin-paclitaxel: NVALT-3 a phase III study. Ann Oncol 2011; January 20 [Epub ahead of print].

[16] Gridelli C, Maione P, Illiano A, et al. Cisplatin plus gemcitabine or vinorelbine for elderly patients with advanced non small-cell lung cancer: the MILES-2P studies. J Clin Oncol 2007; 25: 4663-69.

[17] Rossi A, Galetta D, Gridelli C. Biological prognostic and predictive factors in lung cancer. Oncology 2009; 77 (suppl 1): 90-6.

[18] Crinò L, Cappuzzo F, Zatloukal $\mathrm{P}$, et al. Gefitinib versus vinorelbine in chemotherapy-naive elderly patients with advanced non-small-cell lung cancer (INVITE): A randomized, phase II study. J Clin Oncol 2008; 26: 4253-60.

[19] Jackman DM, Yeap BY, Lindeman NI, et al. Phase II clinical trial of chemotherapy-naïve patients $\geq 70$ years of age treated with erlotinib for advanced non-small-cell lung cancer. J Clin Oncol 2007; 25: 760-6.

[20] Chen Y, Tsai C, Shih J, et al. Phase II randomized trial of erlotinib versus vinorelbine in chemotherapy-naive patients with advanced non-small-cell lung cancer aged $>70$ years in Taiwan. J Clin Oncol 2009; 27(15S): 419s (abstr 8051).

[21] Gridelli C, Morabito A, Gebbia V, et al. Cetuximab and gemcitabine in elderly or adult PS2 patients with advanced nonsmall-cell lung cancer: The cetuximab in advanced lung cancer (CALC1-E and CALC1-PS2) randomized phase II trials. Lung Cancer 2010; 67: 86-92.

[22] Ramalingam SS, Dahlberg SE, Langer CJ, et al. Outcomes for elderly, advanced-stage non small-cell lung cancer patients treated with bevacizumab in combination with carboplatin and paclitaxel: analysis of Eastern Cooperative Oncology Group Trial 4599. J Clin Oncol 2008; 26: 60-5.

[23] Leighl N, Zatloukal P, Mezger J, et al. Efficacy and Safety of Bevacizumab-Based Therapy in Elderly Patients with Advanced or Recurrent Nonsquamous Non-small Cell Lung Cancer in the Phase III BO17704 Study (AVAiL). J Thorac Oncol 2010; 5: 1970-6. 